\title{
Gestión de las emociones, temática importante para los deportistas en situación de competencia
}

\author{
Emotion Management as an Important \\ Topic for Athletes in Competition
}

Andrés Felipe López Cruz ${ }^{1}$

\begin{abstract}
RESUMEN
En este artículo se muestran algunos resultados de una investigación que tiene como objetivo contribuir al fortalecimiento de la gestión de las emociones en los momentos de competencia de los deportistas de fútbol sala, en una institución de educación superior en la ciudad de Bogotá. Se enmarca dentro del deporte como posibilitador hacia el aporte educativo con una mirada pedagógica, asumiéndolo desde tres miradas: la primera es la disciplina deportiva del fútbol sala; por otro lado, el manejo de las emociones por medio de la inteligencia emocional y, como tercer aspecto, generando un pensamiento en el cual la integralidad del ser -que en esta oportunidad es el estudiante universitario- es el objetivo por el cual, en situación de competencia, se debe vislumbrar. Por tal motivo, se desea ofrecer una mirada del tema abordado, sintetizando cada una de las secciones principales para el entendimiento de este artículo, mostrando la necesidad e importancia de cómo fortalecer, por medio de dichas vertientes mencionadas, la gestión de las emociones de los deportistas del fútbol sala, mediante una propuesta pedagógica que logre óptimos resultados ante situaciones de competencia en los torneos universitarios, ilustrando, por medio de los diferentes componentes del mismo, la claridad y el valor hacia este tema tan importante dentro del ámbito deportivo-educativo.
\end{abstract} Palabras clave:

gestión de las emociones, fútbol sala, inteligencia emocional, propuesta pedagógica, inteligencia kinestésica. 


\begin{abstract}
This article presents some of the results of a research study aimed at helping strengthen emotion management indoor soccer players during competition, at an institution of higher education in the city of Bogota. The study is framed within the concept of sport as a facilitator that can contribute to education from a pedagogical point of view, addressing this issue from three perspectives: first, the sports discipline of indoor soccer; second, emotion management through emotional intelligence and, third, generating a way of thinking in which the integral nature of the individual - in this case, the university student - is the objective in competition situations. For this reason, the article looks to offer a look at this topic, synthesizing each of the main sections for the understanding of this article, showing the need and importance of strengthening emotion management in indoor soccer players through the different paths mentioned and a pedagogical proposal that achieves optimal results in university team tournaments and competitions, thus illustrating through the different components thereof, the clarity and value of such an important topic within the field of sports and education.
\end{abstract} Key words: emotion management, indoor soccer, emotional intelligence, pedagogical proposal, kinesthetic intelligence. 


\section{Introducción}

Hay muchos temas que deben atender los deportistas y entrenadores en el contexto de la educación superior, en el cual los estudiantes pueden vincular su participación activa con su proyecto de vida. En el fútbol sala, como en cualquier deporte, hay temas técnicos, tácticos y emocionales que el entrenador debe trabajar de manera conjunta con sus jugadores, para que, de manera progresiva, se obtengan resultados óptimos. Formarse dentro de una disciplina deportiva proporciona a cada individuo una amplitud en su integralidad como ser humano dentro de un círculo social; por ello, en la competencia universitaria se observan diferentes situaciones que forman una situación compleja, teniendo en cuenta aspectos físicos y emocionales y llegando a una cualificación de manera individual y colectiva hacia un objetivo que se desea alcanzar, focalizando la importancia en tres aspectos importantes, como es la disciplina deportiva, el manejo de las emociones y la propuesta pedagógica que se tenga en cuenta como herramienta educativa hacia el logro de objetivos colectivos e individuales encaminados hacia la tan anhelada integralidad.

El presente artículo se centra en la gestión de las emociones desde lo educativo y pedagógico, puesto que se considera un tema importante para todos los deportistas. Así como argumentan Ros Martínez y colaboradores (2013), "el elevado nivel de compromiso personal y la necesidad de rendir siempre al máximo han hecho que la preparación psicológica del deportista se convierta en algo tan necesario como puedan serlo la preparación física o la preparación técnica" (p. 105); no realizar este proceso vulnera al individuo en el momento de la competencia y afecta hacia el desarrollo de sus procesos cognitivos y de aprendizaje.

El deportista debe ser eficaz en el aprendizaje, dado que, por medio de las metodologías impartidas por el docente/entrenador de una forma permanente y con un clima motivacional en el equipo, se llevará el aspecto emocional desde lo individual hacia lo colectivo, formando a cada uno de los individuos, para que así haya una creación en beneficio mutuo para la obtención de resultados como equipo de una manera fructífera, llenando esas necesidades y satisfacciones deportivas que brinda la competición. Balaguer y Duda (2008) sostienen que "la persona, cuando interacciona con su ambiente, necesita sentirse com- 
petente, autónoma y relacionarse con los demás generando un desarrollo en la motivación auto-determinada con un bienestar psicológico individual y colectivo" (p. 124).

Por tal motivo: ¿cómo fortalecer la gestión de las emociones de los deportistas de fútbol sala de la Fundación Universitaria Los Libertadores para que, mediante una propuesta pedagógica, puedan obtener mejores resultados personales y grupales ante situaciones de competencia en los torneos universitarios? Es necesario, en cada deportista, generar una pedagogía hacia la gestión de las emociones en competencia, de forma individual y grupal, haciendo necesario un proceso en el cual se produzca un cambio de perspectiva, lograr un fin en común y así visualizar la verdadera importancia del deporte en el ámbito educativo.

Desde casi una década existen publicaciones en torno al tema desde el ámbito de la psicología deportiva, observando que, en años anteriores, hubo un crecimiento en las publicaciones sobre la inteligencia emocional en el deporte haciendo esto muy atractivo en el ámbito competitivo y educativo, tal y como se muestra a continuación:

Tabla 1.

Publicaciones de trabajos investigativos sobre inteligencia emocional y deporte (2001 - 2012).

\begin{tabular}{ccc}
\hline Año & N $^{\circ}$ e publicaciones & $\%$ \\
\hline 2001 & 1 & 1,66 \\
\hline 2002 & 1 & 1,66 \\
\hline 2003 & 1 & 1,66 \\
\hline 2004 & 2 & 3,33 \\
\hline 2005 & 1 & 1,66 \\
\hline 2006 & 1 & 1,66 \\
\hline 2007 & 5 & 8,3 \\
\hline 2008 & 3 & 5 \\
\hline 2009 & 10 & 16,66 \\
\hline 2010 & 9 & 15 \\
\hline 2011 & 23 & 38,33 \\
\hline 2012 & 3 & 5 \\
\hline Total & 60 & 100 \\
\hline
\end{tabular}

Fuente: Inteligencia emocional y deporte (Ros Martínez et al., 2013, p. 107). 
De esta forma, se considera pertinente generar mayor profundización y una mirada de la gestión de las emociones en el contexto pedagógico y educativo y su incidencia de este campo hacia el desarrollo del ser humano en sus tres dimensiones (cognitivo, físico-emocional), para que se pueda generar, de forma individual y colectiva, la gestión de emociones adquiriendo esas herramientas el deportista en el campo de juego.

Respecto de la gestión de las emociones del estudiante/deportista en relación con la inteligencia emocional, Bisquerra y Pérez (2007) aportan que:

La competencia emocional pone el énfasis en la interacción entre persona y ambiente y, como consecuencia, confiere más importancia al aprendizaje y desarrollo, por lo tanto, tiene unas aplicaciones educativas inmediatas; y la educación emocional, entendida como un proceso educativo continuo y permanente que pretende potenciar el desarrollo de las competencias emocionales como elemento esencial del desarrollo integral de la persona. (pp. 12-13).

Este proceso emocional se reconoce en que la disposición del deportista brinda una identificación en su función dentro del campo de juego, conservando un perfil de inteligencia típico para cada uno de ellos, adquiriendo de esta manera un peso importante el aspecto emocional en la configuración del deportista. Buceta (1998), citado por Del pino Medina y otros (2009), afirma:

Las demandas de rendimiento de cada especialidad deportiva requieren que el deportista funcione, psicológicamente, de una determinada manera, para poder decidir y actuar con la mayor eficacia posible y tener, de esta forma, una opción más favorable de conseguir el éxito en la competición. (p. 5).

Por ello, estos elementos ofrecen una claridad hacia la temática y así brindar un orden al logro de sus aprendizajes, incluyendo comprensión y eficacia a que los conceptos de inteligencia emocional, la competencia emocional y la educación emocional, así como lo plantea Bisquerra (2000), citado por López-Pérez y otros (2008): "la educación emocional es un proceso educativo, continuo y permanente, que pretende potenciar el desarrollo emocional como complemento indispensable del desarrollo cognitivo, constituyendo ambos los ele- 
mentos esenciales del desarrollo de la personalidad integral" (p. 506), evidenciando de esta manera que estos conceptos no son ajenos, dado que tienen cosas en común en el momento de su conceptualización, dándole importancia y la necesidad de que el individuo adquiera dichas competencias emocionales.

La utilización de la gestión de las emociones dentro del contexto educativo y deportivo produce una conducta particular en cada uno de los deportistas, sin desconocer que las emociones intrínsecas y extrínsecas generan una producción colectiva dentro de la unión de dichas conductas, mostrando que ya la emocionalidad no se vivencia de una forma negativa en la sociedad actual y es donde ayuda a la toma de decisiones en algún momento determinado, así como lo expresa Zaccagnini (2004) citado por López-Pérez y otros (2008): “la sociedad actual ha concedido un nuevo papel a las emociones: han dejado de ser algo negativo, a reprimir, para convertirse en un elemento positivo que ayuda a actuar y tomar decisiones $\mathrm{y}$, por tanto, a adaptarse a la vida cotidiana" (p. 504).

Esto se tiene en cuenta y se reconoce al generarse unas conductas emocionales dentro de acciones de los individuos que generan una valoración y una importancia significativa dentro de un contexto; por ello Bisquerra, citado por Salas (2017), afirma:

La emoción se reconoce como un proceso que atraviesa una serie de fases fundamentales, que conllevan a una acción o conducta particular, de este modo se identifica que en un principio se debe producir un evento, ante el cual se da una valoración y posteriormente, se activan tres fases particulares: la fisiológica, la comportamental y la cognitiva, para finalmente producir una predisposición a la acción. (p. 10).

Esto logra que en el deportista se codifiquen aprendizajes que determinan el funcionamiento de talentos especiales, como en esta oportunidad se evidencia el entrelace de la inteligencia cinestésica corporal y las emociones de los deportistas en general, como plantea Gardner (1995), citado por Del Pino Medina y otros (2015): "La cualidad que se atribuye, entre otros, a deportistas y atletas en general, e implica la capacidad de unir cuerpo y mente para lograr el perfeccionamiento en el desempeño físico de manera altamente diferenciada y competente" 
(p. 44), explicando, de esta manera, que el deportista utiliza su corporalidad para estar en competencia, integrando de forma permanente y oportuna la mente y el cuerpo, y formando el control de los aspectos técnicos, tácticos, de forma inteligente, denominando esto como una inteligencia motriz.

Consecuentemente, al plantear esta temática se expone la necesidad de involucrar el tema emocional en el campo deportivo, dado que es importante, dentro de una competencia, que el estudiante/deportista aprenda a adquirir nuevos conocimientos referentes al tema y así lograr de forma progresiva un aprendizaje significativo, formándose en sus campos humanos para la consecución de procesos pedagógicos que ayuden al cumplimiento de los objetivos propuestos hacia su plena integralidad. En este orden de ideas, la gestión de las emociones genera un importante vínculo para el desarrollo del ser humano, tanto a nivel individual como colectivo, en donde se enlaza todo el proceso hacia los estudiantes/deportistas, dado que la competencia genera en cada uno de los seres humanos desniveles en sus conductas de manera individual y colectiva, que hace por momentos esos entornos tensos, pero que con una motivación equilibrada en cada uno de ellos logra que se produzca una armonía, controlando todas las dimensiones humanas dentro de un colectivo deportivo.

\section{Metodología}

El método seleccionado para el desarrollo de este proyecto es un enfoque cualitativo. Según Hernández Sampieri (2006): "El enfoque cualitativo utiliza la recolección y análisis de los datos para afinar las preguntas de investigación o revelar nuevos interrogantes en el proceso de interpretación" (p. 7). De esta manera, el alcance investigativo para comprender esta temática desde la perspectiva pedagógica, dentro del contexto educativo, logra clarificar los niveles por los cuales las metodologías, las didácticas y las estrategias utilizadas exploran sus debilidades y potencian las habilidades de cada uno de los individuos.

El tipo de investigación que a este proyecto concierne es Investigación Acción (IA), comprendiendo que tiene como finalidad brindar una comprensión y una solución a la problemática que se esté abordando, aclarando que no solo es un proceso de solución de problemas, sino también el lograr modificación de las prácticas y modelos ya antes 
trabajados; así como lo menciona McKernan, citado por Hernández Sampieri (2014):

Implica la total colaboración de los participantes en la detección de necesidades (ya que ellos conocen mejor que nadie la problemática a resolver), el involucramiento con la estructura a modificar, el proceso a mejorar, las prácticas que requieren cambiarse y la implementación de los resultados del estudio. (pp. 496-497).

Esta investigación aporta a la mejoría de las prácticas y procesos, brindando una mayor solidez hacia el análisis y la conceptualización en términos de la investigación educativa de la gestión de las emociones. Este tipo de investigación hace que el tema abordado se vuelva práctico y participativo, implementando un desarrollo en el plan de acción deseado para mejorar y brindar cambios positivos hacia el nivel de vida de los individuos de manera integral y educativa frente al ámbito deportivo.

Para esto, a partir del desarrollo teórico se generó una propuesta pedagógica hacia los estudiantes/deportistas para trabajar capacidades físicas propias del deporte y la gestión de emociones con el equipo que, en particular, se evidencien en situación de competencia; finalizando con la pertinencia de la estructura de dicha propuesta con la muestra mencionada. En este instrumento final se analizó lo que expresaron los estudiantes/deportistas en un taller realizado, respecto de los aportes del equipo de fútbol sala de la Fundación Universitaria Los Libertadores. Se pretendió, por medio de éste, concientizar a los estudiantes de la importancia de la emocionalidad para fomentar la participación en el fortalecimiento de la propuesta hacia el mejoramiento del rendimiento deportivo de forma individual y colectiva.

Este taller se realizó por medio del siguiente procedimiento: en primer lugar, se explicaron los resultados del test diagnóstico; luego se realizó la explicación a los estudiantes/deportistas de la propuesta pedagógica diseñada y, por último, se entregó un cuestionario a cada persona, con el fin de que ellos opinaran sobre la pertinencia de la propuesta pedagógica.

Esta estructura de la propuesta se diseñó para la muestra seleccionada, en la cual se examina por la temática de la gestión de las emociones y su comprensión de la inteligencia emocional. Fue aplicada a una muestra constituida por 26 estudiantes/deportistas que conforman los 
equipos masculino y femenino que estudian en Bogotá en una institución de educación superior.

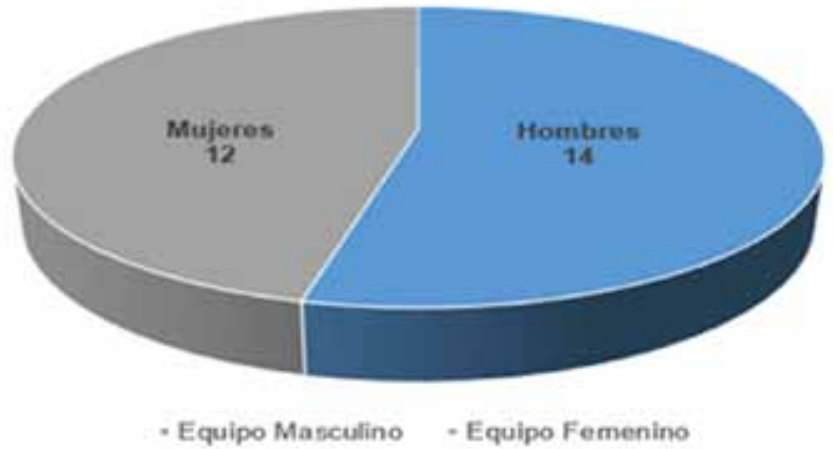

Figura 1. Distribución de la muestra.

Fuente: elaboración propia (2019).

Como se observa en la figura 1, la muestra contempla 26 estudiantes: 14 del género masculino y 12 del género femenino. El instrumento utilizado fue un cuestionario; las preguntas indagaron sobre emociones y sentimientos de cada uno de los individuos que conforman la muestra de estudio.

Se tuvo en cuenta el pensar y el sentir de los estudiantes/deportistas y de los entrenadores de la institución universitaria, exponiendo de forma general que hay puntos de vista muy importantes y paralelos en lo que respecta al tema en mención. Notan los entrenadores que, en el momento de la competencia, sus deportistas gestionan sus emociones de forma equívoca en la práctica y, aunque son conscientes de ello, no lo modifican al momento de jugar.

\section{Resultados}

Este estudio reveló que, ante las falencias en la gestión de las emociones en situación de competencia, en el ámbito deportivo se tiene plena conciencia en la actualidad de que la emocionalidad juega un papel importante en el individuo como actor individual y social, pero que en el momento de la práctica no se hace evidente la producción de las emociones. Adicionalmente se quiere resaltar que, al momento de la realización de esta práctica investigativa, se permitió un acercamiento 
entre los estudiantes/deportistas y los docentes, arrojando de esta forma resultados y opiniones hacia el tópico emocional en sus deportistas, brindando con esto aportes dentro del proyecto de investigación, para que se logre en los estudiantes un proceso netamente formativo hacia la gestión de la emocionalidad en el contexto deportivo.

Se observa en la cultura una difícil comprensión de las emociones en los seres humanos, para quienes no es fácil conectar la conciencia de las emociones con el lugar de la persona y del otro, lo cual acarrea una ruptura en el proceso mental e implicaciones en el esfuerzo emocional, tal como lo expresan Fernández Berrocal y Pacheco (2002):

Para comprender los sentimientos de los demás debemos empezar por aprender a comprendernos a nosotros mismos, cuáles son nuestras necesidades y deseos, qué cosas, personas o situaciones nos causan determinados sentimientos, qué pensamientos generan tales emociones, cómo nos afectan y qué consecuencias y reacciones nos provocan. (p. 4).

El cuerpo de la propuesta contiene ejercicios para trabajar con el equipo deportivo, teniendo en cuenta las siguientes fases en el individuo: capacidad física, coordinativa, operativa (cognición), socioemotiva y autocreativa, abordando capacidades físicas propias del deporte y la gestión de emociones que, en particular, se evidencien en situación de competencia.

\begin{tabular}{ll}
\hline Contenido propuesto & Objetivo \\
\hline $\begin{array}{l}\text { Ejercicios de agilidad y } \\
\text { manejo de la emoción } \\
\text { tensión/estrés. }\end{array}$ & $\begin{array}{l}\text { Trabajar la gestión emocional del estrés y la } \\
\text { capacidad física de la agilidad para realizar } \\
\text { acciones motoras en el menor tiempo posible } \\
\text { utilizando la comunicación como herramienta } \\
\text { de orden grupal. }\end{array}$ \\
\hline $\begin{array}{l}\text { Ejercicios de } \\
\text { coordinación y manejo } \\
\text { de la emoción de la } \\
\text { ansiedad. }\end{array}$ & $\begin{array}{l}\text { Potenciar el manejo de la ansiedad y la } \\
\text { capacidad física complementaria de la } \\
\text { coordinación, a través de la relación entre } \\
\text { músculos y sentidos, generando así acciones } \\
\text { diversas, para manejar de esta manera la actitud } \\
\text { del individuo en el ejercicio a realizar. }\end{array}$ \\
\hline $\begin{array}{l}\text { Ejercicios de velocidad } \\
\text { y manejo de la emoción } \\
\text { de la cohesión. }\end{array}$ & $\begin{array}{l}\text { Fomentar el manejo de la cohesión junto a la } \\
\text { capacidad física de la velocidad, para realizar } \\
\text { acciones motoras en el menor tiempo posible } \\
\text { utilizando la comunicación como herramienta } \\
\text { de orden grupal. }\end{array}$ \\
\hline
\end{tabular}




\begin{tabular}{ll}
\hline $\begin{array}{l}\text { Ejercicios de fuerza } \\
\text { explosiva y manejo } \\
\text { de la emoción de la } \\
\text { frustración. }\end{array}$ & $\begin{array}{l}\text { Propiciar el manejo de la frustración en fusión de } \\
\text { la capacidad física de la fuerza, para minimizar } \\
\text { el sentimiento prolongado de dicha emoción. }\end{array}$ \\
\hline $\begin{array}{l}\text { Ejercicios de circuitos } \\
\text { variados y manejo de } \\
\text { la emoción del trabajo } \\
\text { en equipo (cultura de } \\
\text { equipo). }\end{array}$ & $\begin{array}{l}\text { Medir la capacidad de acción de los estudiantes } \\
\text { a diferentes estímulos coordinativos, de } \\
\text { equilibrio y de resistencia, para aumentar } \\
\text { los niveles perceptivos y óseo-musculares, } \\
\text { fomentando la confianza y el compañerismo } \\
\text { dentro del grupo. }\end{array}$ \\
\hline $\begin{array}{l}\text { Análisis reflexivo hacia } \\
\text { el beneficio colectivo. }\end{array}$ & $\begin{array}{l}\text { Observar de manera crítica una grabación de } \\
\text { un partido de competencia para analizar de } \\
\text { manera constructiva el beneficio de la cohesión } \\
\text { de grupo. }\end{array}$ \\
\hline
\end{tabular}

Fuente: Elaboración propia (2019).

Con la implementación de esta propuesta pedagógica para el desarrollo se generarán en el equipo deportivo muchos puntos positivos hacia la adquisición de nuevos logros colectivos y, además de ello, se gana un clima de confianza individual y de equipo, mostrando un desempeño más sólido y realista hacia el seguimiento de criterios y sistemas de manejo deportivo y pedagógico.

Seguido a esto y de acuerdo con lo encontrado en el transcurso de este objetivo, el cual es analizar la pertinencia de la propuesta pedagógica por parte de los estudiantes/deportistas de los grupos representativos de fútbol sala de la Fundación Universitaria Los Libertadores, se presenta lo siguiente:

Tabla 2.

Aporte e importancia de la propuesta pedagógica.

\begin{tabular}{lc}
\hline \multicolumn{1}{c}{ Subcategorías emergentes } & Frecuencia \\
\hline Totalmente de acuerdo & $76,5 \%$ \\
Algo de acuerdo & $23,5 \%$ \\
Nada de acuerdo & $0,0 \%$ \\
\hline
\end{tabular}

Fuente: Elaboración propia (2019).

Analizando lo que se evidencia en la tabla 2, de manera general y en contraste con los teóricos, se evidencia la importancia en el aporte del diseño de la propuesta pedagógica hacia el beneficio de la gestión de las emociones en situación de competencia, expresado por los estu- 
diantes/deportistas de futbol sala de los grupos representativos, brindando relevancia en el aspecto educativo y formativo de forma individual llegando a lo colectivo, tal y como lo expresa Marveya (2006): “La educación ofrece a la persona una formación individual, pero siempre con proyección social" (p. 39), en donde se quiere lograr con el diseño de esta propuesta el aporte del manejo emocional en el deporte de manera formativa hacia un objetivo grupal.

La tabla 3 muestra los resultados de los estudiantes/deportistas en el tema de la claridad en los procedimientos y objetivos de la propuesta, con base en respuestas dadas por los hombres y mujeres de la muestra:

Tabla 3.

Claros procedimientos y objetivos de la propuesta pedagógica.

\begin{tabular}{lc}
\hline \multicolumn{1}{c}{ Subcategorías emergentes } & Frecuencia \\
\hline Totalmente de acuerdo & $76,5 \%$ \\
Algo de acuerdo & $23,5 \%$ \\
Nada de acuerdo & $0,0 \%$ \\
\hline
\end{tabular}

Fuente: Elaboración propia (2019).

En el análisis de esta figura, un 76,5\% expresó que, al escuchar la estructura de la propuesta, tuvo claridad y encontró importante la propuesta pedagógica hacia el aprendizaje de la gestión de las emociones en situación de competencia, lo cual hace reflexionar, por parte de los deportistas, en la pertinencia de la misma hacia el aprender a manejar la emocionalidad en el ámbito deportivo y así disminuir las falencias encontradas y lograr un hábito en la utilización de las estrategias para obtener una regularidad en sus acciones técnicas, físicas, tácticas y emocionales. Por ello, y así como lo plantea Sacristán Roa (2010): “La práctica se somete a las estructuras que ella misma crea y que por ello dan continuidad y regularidad a las acciones humanas. Son generadoras de hábitos que a su vez las prefiguran" (p. 28).

La tabla 4 exhibe los resultados dados por hombres y mujeres de la muestra acerca de la importancia de la propuesta en el deporte: 
Tabla 4.

Importancia de la propuesta pedagógica en el deporte.

\begin{tabular}{lc}
\hline \multicolumn{1}{c}{ Subcategorías emergentes } & Frecuencia \\
\hline Totalmente de acuerdo & $70,6 \%$ \\
Algo de acuerdo & $29,4 \%$ \\
Nada de acuerdo & $0,0 \%$ \\
\hline
\end{tabular}

Fuente: Elaboración propia (2019).

Analizando la tabla 4, un 70,6\% está totalmente de acuerdo con la importancia de la propuesta, junto a un $29,4 \%$ que se encuentra algo de acuerdo con esa importancia.

En la tabla 5 se muestran los resultados relativos al aporte de la propuesta en el rendimiento, encontrando en los estudiantes/deportistas lo siguiente:

Tabla 5.

Afecta lo emocional en el rendimiento.

\begin{tabular}{lc}
\hline \multicolumn{1}{c}{ Subcategorías emergentes } & Frecuencia \\
\hline Totalmente de acuerdo & $70,5 \%$ \\
Algo de acuerdo & $23,5 \%$ \\
Nada de acuerdo & $6,0 \%$ \\
\hline
\end{tabular}

Fuente: Elaboración propia (2019).

Analizando la tabla, hay un valor significativo del $76,5 \%$ de estudiantes al escuchar la estructura de la propuesta pedagógica. Ellos están totalmente de acuerdo con que el aporte de la propuesta es importante. Esta relevancia en el aporte formativo de la propuesta pedagógica, hace reflejar en los resultados la importancia que expresan los estudiantes/deportistas respecto de la unión entre lo físico (deporte) y lo mental (emocional), formando de esta manera un desarrollo paralelo hacia la práctica, lo que hace reafirmar lo planteado por Goleman (2008) cuando expresa: "La dicotomía entre lo emocional y lo racional se asemeja a la distinción popular existente entre el corazón y la cabeza" (p. 43), porque el ámbito racional hace que lo emocional se haga de una manera equilibrada.

Por ello, para finalizar este análisis de los resultados, de manera general se evidencia la importancia del aporte de diseño de la propuesta pedagógica hacia el beneficio de la gestión de las emociones en situa- 
ción de competencia, expresado por los estudiantes/deportistas de futbol sala de los grupos representativos, brindando relevancia en el aspecto educativo y formativo de forma individual llegando a lo colectivo, en donde se quiere lograr, con el diseño de esta propuesta, el aporte del manejo emocional en el deporte de manera formativa hacia un objetivo grupal.

\section{Conclusiones}

El objetivo de este escrito ha sido mostrar que el trabajo de la gestión de las emociones en el contexto educativo es un factor de constructo útil, bien fundamentado y brinda la comprensión y generación de un aprendizaje que conduzca a "desaparecer" dentro de estas prácticas deportivas las falencias que los seres humanos experimentan dentro del ámbito emocional, y en cambio se apropien de prácticas fundamentadas desde el aspecto pedagógico que lleven a cada uno de los estudiantes/deportistas, con el acompañamiento del docente/entrenador, al manejo de sus emociones en situación de competencia, lo cual implica hacer cambios e innovación en el trabajo tradicional de entrenadores, para no quedarse en el trabajo físico, sino también involucrar estrategias formativas que permitan gestionar sus emociones y tener un comportamiento que incida en mejores resultados individuales y de equipo, dentro de un espacio deportivo y competitivo.

La temática abordada ha dado muchos giros en torno a la psicología, pero con esta investigación se quiere lograr que el aspecto pedagógico se evidencie desde el aprendizaje de la gestión de las emociones en los estudiantes/deportistas de una manera formativa, dado que este aspecto logrará en el individuo una regulación de las propias emociones. Así como lo expresan Mayer y Salovey, citado por Extramera y Fernández Berrocal (2008): "La regulación consciente de las emociones para lograr un crecimiento emocional e intelectual. Con objeto de conseguir una efectiva regulación de nuestras propias emociones y de las personas que conforman nuestro entorno social" (p. 102). De esta forma, se generará una eficacia en el manejo de las emociones de las personas en situación de competencia. La propuesta se estructura desde el concepto del "hexágono pedagógico", e incluye un componente pedagógico y uno didáctico, tal como se presenta en la figura 2 : 


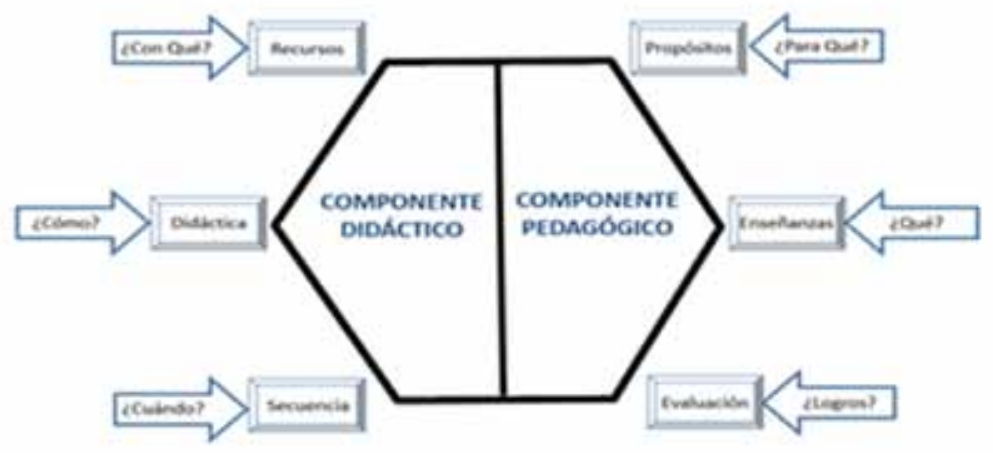

Figura 2: Hexágono Pedagógico: Propuesta pedagógica Conceptual. Fuente: elaboración propia (2019).

El diseño de la propuesta pedagógica hacia la gestión de las emociones de los deportistas de futbol sala en situación de competencia, y los resultados obtenidos durante todas las fases de la investigación, lleva al investigador a corroborar que, a través del diseño propuesto, desde el ámbito formativo aporta a la disminución de las falencias en el manejo emocional, pues, por medio de las estrategias pedagógicas, los estudiantes/deportistas logran transformar sus conductas, permitiendo así la armonía en el trabajo en equipo y canalizar el autocontrol dentro del entorno deportivo.

Justo es decir que, a la mayoría de los estudiantes les gusta mucho el deporte que practican, pero se nota la carencia en el aprendizaje emocional de forma pedagógica y formativa, saliendo a relucir las falencias en la gestión de las emociones. Se observó que, durante los momentos de escucha hacia su docente/entrenador, se logró de manera intermitente la utilización de las herramientas de forma óptima, dado que lo emocional los logra desequilibrar y bajar su rendimiento deportivo.

Ante estas falencias, se observa que en el ámbito deportivo se tiene plena conciencia en la actualidad de que la emocionalidad juega un papel importante en el individuo como actor individual y social, pero no se le da el fortalecimiento necesario en la ruta pedagógica y educativa hacia un aprendizaje significativo de los mismos partícipes; como docentes/entrenadores debemos tener claro el foco de impor- 
tancia que la educación y la pedagogía puede generar en el aspecto emocional, dentro del contexto deportivo hacia un proceso formador en el cual uno se debe involucrar, llevando de esta manera a un aprendizaje significativo y teniendo en cuenta que, en el contexto universitario, los estudiantes son fluctuantes y se producen cambios constantes en los equipos.

Adicionalmente, se logró que los estudiantes adquirieran conciencia de la importancia de la gestión de las emociones a través de la comprensión, la escucha del diseño de la propuesta pedagógica y los aportes con los entrenadores y los estudiantes para enriquecer la propuesta pedagógica diseñada, para obtener en los estudiantes un proceso netamente formativo hacia la gestión de la emocionalidad en el contexto deportivo. Con esto se quiere resaltar que, al momento de la realización de esta práctica investigativa, se permitió un acercamiento entre los estudiantes/deportistas y los docentes, arrojando resultados y aportes significativos hacia el proyecto de investigación.

\section{Referencias}

Balaguer, I. \& Duda, J. (2008). Apoyo a la autonomía, satisfacción de las necesidades, motivación y bienestar en deportistas de competición. Revista de Psicología del deporte. España: Universidad Autónoma de Barcelona.

Bisquerra, R. y Pérez N. (2007). Las competencias emocionales. España: Universidad de Barcelona.

Del Pino Medina, J., Gómez Milán, E., Moreno Ríos, S. Gálvez García, G. \& Mula Pérez, F. (2015). Inteligencias múltiples y rendimiento en fútbol. Apunts Educación Física y Deportes, (95), 5-13.

Extramera, N. \& Fernández- Berrocal, P. (2008). La inteligencia emocional en el contexto educativo: hallazgos científicos de sus efectos en el aula. Revista de Educación.

Fernández-Berrocal, P. \& Pacheco, N. (2002). La inteligencia emocional como una habilidad esencial en la escuela. Revista Iberoamericana de Educación (ISSN: 1681-5653). Universidad de Málaga. España.

Goleman, D. (2008). Inteligencia Emocional. Barcelona: Editorial Kairós. Hernández Sampieri, R. (2006). Metodología de la investigación. Cuarta Edición. 
López-Pérez, B., Fernández-Pinto, I. \& Márquez-González, M. (2008). Educación emocional en adultos y personas mayores. Electronic Journal of Research in Educational Psychology, 6(2), 501-522.

Marveya, E. (2006). Didáctica Integrativa y el proceso de aprendizaje. México: Editorial Trillas.

Ros Martínez, A., Moya-Faz, F. \& Garcés de Los Fayos, E. (2013). Inteligencia emocional y deporte: situación actual del estado de la investigación. Psicología del Deporte, 13(1), 105-112.

Sacristán Roa, O. (2010). Prácticas deportivas exitosas en el centro javeriano de formación deportiva en su programa entrenamiento deportivo de facultades. Tesis de especialización. Bogotá. Colombia. Recuperado de https://repository. javeriana.edu.co/bitstream/handle/10554/419/edu48. pdf? sequence $=1$ \&isAllowed $=\mathrm{y}$

Salas, A. (2017). Desarrollo de competencias emocionales en estudiantes entre los 9 y 12 años de edad en un colegio privado de la ciudad de Bogotá. Colombia: Universidad del Rosario. 